\title{
Desarrollo de habilidades comunicativas en lactantes con síndrome de Down posterior a capacitaciones sistematizadas en comunicación gestual
}

\section{Development of communicative abilities in infants with Down syndrome after systematized training in gestural communication}

\author{
Katherina Linn ${ }^{\mathrm{a}}$, Fabiana Sevilla ${ }^{\mathrm{b}, \mathrm{c}}$, Valeria Cifuentes ${ }^{\mathrm{b}, \mathrm{c}}$, M. Ignacia Eugenin ${ }^{\mathrm{d}}$, \\ Bernardita Río $^{c, e}$, Jaime Cerda ${ }^{\mathrm{f}}$, Macarena Lizama ${ }^{\mathrm{c}}$ \\ aDivisión de Pediatría, Escuela de Medicina, Pontificia Universidad Católica de Chile \\ bFonoaudióloga \\ 'Centro UC Síndrome de Down \\ 'Red de Salud UC CHRISTUS \\ eKinesióloga \\ ‘Departamento de Salud Pública, Pontificia Universidad Católica de Chile
}

Recibido: 20 de marzo de 2018; Aceptado: 27 de noviembre de 2018

\begin{abstract}
Resumen
La comunicación gestual, entendida como el uso de gestos no verbales antes de la aparición de la palabra, es una fortaleza en niños con síndrome de Down (SD). Objetivo: Describir conductas de desarrollo comunicativo en niños con SD, previo y posterior a capacitaciones en comunicación gestual, basadas en talleres de "Señas, palabras y juegos" del programa Baby Signs ${ }^{\circledR}$. Sujetos y Método: Estudio prospectivo de niños con SD entre 18 y 22 meses de edad cognitiva, a los cuales se les realizó capacitaciones en comunicación gestual según la metodología Baby Signs, evaluando habilidades comunicativas a través del inventario McArthur adaptado para niños con SD (Inventario de desarrollo comunicativo, CDI-SD), analizando los puntajes antes y 3 meses después de la intervención. Los ítems evaluados corresponden a: Comprensión temprana, Comprensión de las primeras frases, Comenzando a hablar, Lista de vocabulario y Uso descontextualizado del lenguaje (parte 1) y a Gestos totales, tempranos y tardíos (parte 2). Resultados: 21 niños completaron los talleres, con una media de edad cronológica de 27,5 meses y 19,8 meses de edad cognitiva. El 29\% de los participantes aumentaron sus puntajes en comprensión de frases, el $62 \%$ en producción de vocabulario con gestos, el $33 \%$ mejora la comprensión de vocabulario, el 57\% perdió los gestos tempranos y el $43 \%$ aumentó la producción de gestos tardíos. Conclusiones: Las capacitaciones en comunicación gestual favorecen el desarrollo de habilidades comunicativas en un grupo de niños con SD, principalmente en la comprensión inicial y producción de gestos. Existe importante variabilidad interindividual, por lo que es necesario considerar las recomendaciones niño a niño.
\end{abstract}

Palabras clave: Síndrome de Down; gestos; comunicación no verbal; ayudas de comunicación; discapacidad 


\section{Abstract}

Introduction: Gestural communication, understood as the use of non-verbal gestures before the word appears, is a strength in children with Down syndrome (DS). Objective: To describe communication development behaviors in children with DS, before and after gestural communication training, based on the "Signs, words and games" workshops of the Baby Signs ${ }^{\circledR}$ program. Subjects and Method: Prospective study of children with DS between 18 and 22 months of cognitive age, who were trained in gestural communication according to the "Baby Signs ${ }^{\circledR}$ " methodology, evaluating communication skills through the MacArthur inventory adapted for children with DS (Communicative Development Inventories, CDI-DS), analyzing the scores before and three months after the intervention. The evaluated items were: Early comprehension, First sentences comprehension, Starting to speak, Vocabulary list, and Decontextualized language use (part 1) and total, early and late gestures (part 2). Results: 21 children completed the workshops, with an average chronological age of 27.5 months and 19.8 months of cognitive age. $29 \%$ of the participants increased their scores in sentence comprehension, $62 \%$ in vocabulary production with gestures, $33 \%$ improved in vocabulary comprehension, 57\% lost early gestures, and $43 \%$ increased late gestures production. Conclusions: Gestural communication training favors the communication skills development in a group of children with DS, mainly in the initial understanding and gesture production. There is important inter-individual variability, therefore is necessary to consider child to child recommendations.

\section{Keywords:}

Down syndrome;

gestures;

non-verbal

communication;

communication aids;

disability

\section{Introducción}

La comunicación gestual es el uso de gestos no verbales simples, para la representación de diferentes elementos ${ }^{1,2}$, y surge como puente antes de la aparición de la palabra ${ }^{3}$. Se describe en la década de los ochenta, a partir de observaciones de las doctoras en psicología Linda Acredolo y Susan Goodwyn, sobre bebés que usaban gestos para reemplazar palabras que no podían pronunciar. Acredolo y Goodwyn demostraron que la exposición temprana a comunicación con gestos promueve el desarrollo del lenguaje expresivo y comprensivo, y aumenta las tareas fonéticas y de sintaxis. Otros estudios han demostrado que el desarrollo del lenguaje a través de gestos, previo el desarrollo del habla en niños con desarrollo típico, fomenta la interacción padres-hijos, disminuye los niveles de frustración tanto en el niño como en sus padres ${ }^{5}$, permite expresar estados emocionales en el niño en etapa preverbal ${ }^{6}$, estimula el desarrollo cognitivo ${ }^{1}$ y favorece la autorregulación ${ }^{7}$.

Los niños con síndrome de Down (SD) presentan retraso en el desarrollo psicomotor, discapacidad cognitiva de grado variable y especialmente retraso en el desarrollo del lenguaje con una disociación entre las habilidades receptivas y las expresivas, teniendo mayor dificultad en la comunicación expresiva, llegando incluso a la ausencia de palabras ${ }^{8}$. Considerando lo antes descrito, el interés social y el uso de gestos pre-lingüísticos emerge como una de las principales oportunidades para la interacción con el medio, ${ }^{9,10}$.

Diversos autores describen que la comunicación gestual es uno de los puntos fuertes de los niños con $\mathrm{SD}^{11,12}$, siendo el vocabulario productivo equiparable al de niños con desarrollo típico si se toman en cuenta los gestos ${ }^{13}$. Sin embargo, a la fecha, no hay datos sobre intervenciones en el desarrollo de comunicación gestual en esta población.

El programa Baby Signs ${ }^{\circledR}$ surge a partir de las investigaciones realizadas por Acredolo y Goodwyn, como una metodología para enseñar a papás, cuidadores y sus hijos, la incorporación de gestos simples, que complementan la comunicación. Este programa consta de diferentes modalidades, tanto para familias como para instructores en comunicación gestual, y se basa en talleres presenciales y uso de material didáctico, como tarjetas y canciones para la enseñanza y aprendizaje de gestos.

Considerando lo antes descrito, el objetivo principal de este estudio es describir las conductas de desarrollo comunicativo en niños con $\mathrm{SD}$, previo y posterior a la realización de capacitaciones en comunicación gestual, basadas en la metodología de "Señas, palabras y juegos" del programa Baby Signs ${ }^{\circledR}$.

\section{Sujetos y Método}

Estudio prospectivo de tipo descriptivo, realizado entre enero y noviembre de 2017.

\section{Población a intervenir}

Se invitó a participar a niños entre 24 y 30 meses de edad cronológica con diagnóstico de SD. El rango etario fue establecido en forma arbitraria estimando obtener una muestra de niños entre 18 y 22 meses de edad cognitiva. Estos fueron convocados a través de una invitación abierta por correo electrónico, redes sociales del Centro UC SD, y por el equipo de NANEAS UC. 
El cálculo de edad cognitiva fue necesario para poder aplicar en forma adecuada el inventario de desarrollo comunicativo (CDI) adaptado a población con SD (CDI-SD), cuyos resultados son interpretables según edad mental del niño y no cronológica.

La elección del rango de edad cognitiva del grupo a intervenir fue determinada a partir de la estimación de edad en que los niños con desarrollo típico ya han adquirido los primeros gestos y van adquiriendo gestos en forma habitual ${ }^{14}$.

\section{Instrumento de evaluación de comunicación}

Se utilizó el CDI-SD previo y posterior a la intervención. El CDI es un instrumento ampliamente utilizado para la evaluación del lenguaje en niños con desarrollo típico y evalúa primeras palabras, gestos y gramática. Su versión original está traducida a diversos idiomas y validada al español por Jackson-Maldonado $^{15}$. El CDI-SD es el instrumento CDI previamente validado al español, adaptado para niños con SD. La adaptación y validación fue realizada por Galeote, en población española e hispanoparlante ${ }^{16}$, donde las principales adaptaciones se basan en: utilización de un único inventario que abarca el rango etario de 8 a 30 meses (el original lo separa en dos grupos etarios); no se considera la edad cronológica, sino la edad de desarrollo evaluada mediante la Escala de Desarrollo Psicomotor de la Primera Infancia Brunet-Lezine-Revisada $\mathrm{u}$ otros tests comparables, como el Test de Bayley; y en la evaluación de vocabulario se analiza comprensión, producción y gesticulación de la palabra. La validación realizada por Galeote mostró una correlación estadísticamente significativa en los segmentos de producción de vocabulario, vocabulario receptivo y la confiabilidad para producción y comprensión de vocabulario también se describe como fuerte y estadísticamente significativa, al evaluar test- retest con 3 meses de diferencia ${ }^{16}$.

El estudio consistió en tres fases (esquema de metodología en Figura 1).

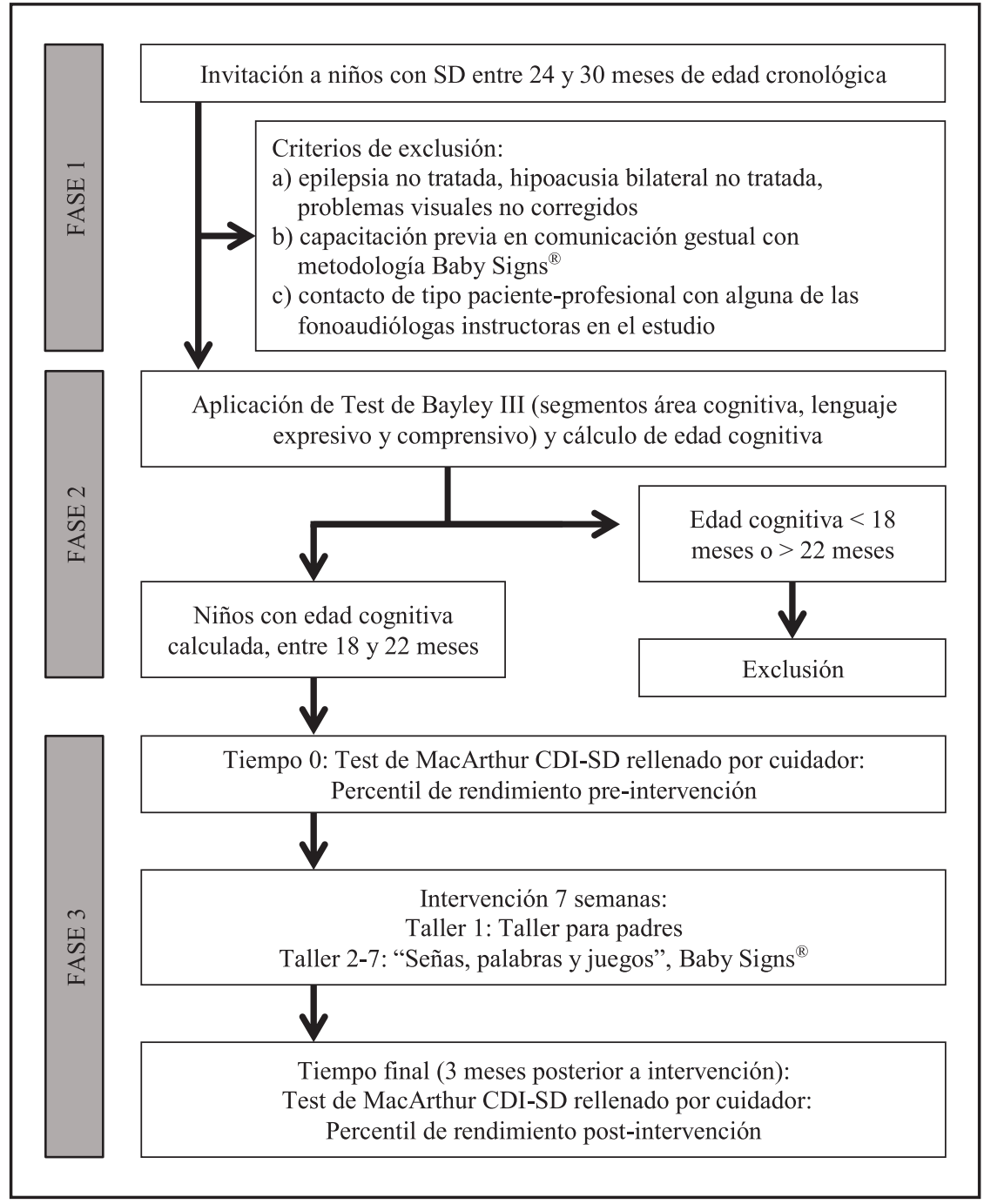

Figura 1. Esquema cronológico sobre fases y metodología del estudio. 
Fase 1: Reclutamiento de población interesada. Los cuidadores que manifestaron interés en participar cuyos niños cumplían criterios de edad cronológica, fueron invitados para evaluación y determinación de edad cognitiva. Fueron excluidos aquellos niños con: a) diagnóstico confirmado de epilepsia no tratada, hipoacusia bilateral (potenciales evocados auditivos de tronco con ondas $\mathrm{V}$ mayores a $50 \mathrm{~dB}$ ) no tratada, o problemas visuales no corregidos (cataratas congénitas no operadas); b) antecedentes de capacitación previa en comunicación gestual con metodología Baby Signs ${ }^{\circledR}$ y c) antecedente de contacto de tipo paciente-profesional con alguna de las fonoaudiólogas instructoras en el estudio.

Fase 2: Selección de población objetivo. A aquellos niños que cumplían con criterios para participar se les aplicó test de Bayley $\mathrm{III}^{17}$ para determinar su edad cognitiva. El grupo objetivo a intervenir fue de niños con edad cognitiva entre 18 y 22 meses. El test de Bayley III fue realizado por tres profesionales capacitados, quienes aplicaron los segmentos de área cognitiva, lenguaje receptivo y expresivo. La edad cognitiva fue calculada según el puntaje de escala cognitiva del test de Bayley
III. Los participantes con edad cognitiva entre 18 y 22 meses fueron convocados a participar de la evaluación y capacitación en comunicación gestual. A todos los cuidadores de los niños a quienes se les aplicó el test de Bayley III, se les entregó un informe de evaluación, fueran o no a participar de la Fase 3 del proyecto.

Fase 3: Evaluación y capacitación. A cada niño seleccionado y su respectivo cuidador significativo, se les aplicó:

1. Evaluación inicial: evaluación del desarrollo comunicativo de cada participante previo al inicio de las capacitaciones, a través del CDI-SD ${ }^{18}$, autorizado por el autor para su uso en investigación y autoaplicado por los cuidadores ${ }^{19}$.

En la tabla 1 se encuentra la descripción de los ítems del CDI-SD que fueron evaluados. Para los ítems 1B, 1D, y gestos, el informe del test entrega los resultados en percentiles de rendimiento. Para los ítems 1A, $1 \mathrm{C}$ y $1 \mathrm{E}$ se entrega resultado descriptivo, calculando el porcentaje de respuestas positivas y se dispone de tablas comparativas para porcentaje de respuestas estándar según grupo de edad cognitiva (tabla 1).

Se solicitó que el formulario fuese contestado por

Tabla 1. Descripción de Inventario de desarrollo comunicativo adaptado por Galeote para niños con síndrome de Down (CDI-SD)

\begin{tabular}{|c|c|c|}
\hline Primeras palabras & Definición & Expresión de resultados \\
\hline $\begin{array}{l}\text { A. Primeros signos de } \\
\text { comprensión: comprensión } \\
\text { temprana }\end{array}$ & $\begin{array}{l}\text { Incluye una serie de preguntas sobre palabras que los niños suelen com- } \\
\text { prender en los comienzos del aprendizaje del lenguaje: a) responde al } \\
\text { nombre, b) responde al no y c) busca cuando llaman a papá o mamá. }\end{array}$ & $\begin{array}{l}\text { Porcentaje de respuestas afirma- } \\
\text { tivas según edad cognitiva, com- } \\
\text { paradas con referencia de Galeote }\end{array}$ \\
\hline $\begin{array}{l}\text { B. Comprensión de las } \\
\text { primeras frases }\end{array}$ & $\begin{array}{l}\text { Incluye una serie de preguntas sobre frases simples y muy familiares que } \\
\text { los niños suelen comprender en los comienzos del aprendizaje. Ejemplo: } \\
\text { "A dormir", "dame un beso". }\end{array}$ & $\begin{array}{l}\text { Percentil de rendimiento según } \\
\text { edad cognitiva }\end{array}$ \\
\hline $\begin{array}{l}\text { C. Comenzando a hablar: } \\
\text { los inicios de la producción }\end{array}$ & $\begin{array}{l}\text { Evalúa el modo en que los niños comienzan a producir sus primeras pala- } \\
\text { bras (grado de imitación de las frases y palabras que escuchan, denomina- } \\
\text { ción de los objetos que están a su alrededor. a) ¿Imita palabras o parte de } \\
\text { frases?, b) ¿nombra elementos que ve?". }\end{array}$ & $\begin{array}{l}\text { Porcentaje de respuestas afirma- } \\
\text { tivas según edad cognitiva, com- } \\
\text { paradas con referencia de Galeote }\end{array}$ \\
\hline $\begin{array}{l}\text { D. Lista de vocabulario*: } \\
\text { comprende, comprende } \\
\text { y dice, comprende y } \\
\text { gesticula }\end{array}$ & $\begin{array}{l}\text { Lista de } 651 \text { palabras divididas en } 21 \text { categorías. Evaluando en cada una } \\
\text { de ellas comprensión y producción a) comprende y b) comprende y dice. } \\
\text { Además evalúa la utilización de gestos para referirse a los distintos ítems } \\
\text { de vocabulario incluidos en el inventario c) comprende y gesto. }\end{array}$ & $\begin{array}{l}\text { Percentil de rendimiento según } \\
\text { edad cognitiva }\end{array}$ \\
\hline $\begin{array}{l}\text { E. Uso descontextualizado } \\
\text { del lenguaje }\end{array}$ & $\begin{array}{l}\text { Evalúa el grado en que los niños emplean el lenguaje para referirse a } \\
\text { objetos, personas o eventos que no están presentes, a) sucesos que han } \\
\text { ocurrido en el pasado, b) en el futuro, c) producción de objeto ausente d) } \\
\text { comprensión de objeto ausente y e) dueño ausente. }\end{array}$ & $\begin{array}{l}\text { Porcentaje de respuestas afirma- } \\
\text { tivas según edad cognitiva, com- } \\
\text { paradas con referencia de Galeote }\end{array}$ \\
\hline Gestos & $\begin{array}{l}79 \text { gestos y acciones divididos en } 6 \text { categorías: (1) primeros gestos, ( } 2 \text { ) jue- } \\
\text { gos con adultos y rutinas, ( } 3 \text { ) acciones con objetos, (4) jugar a ser adulto, } \\
\text { (5) imitación de otros tipos de actividades de los adultos y (6) acciones con } \\
\text { un objeto en lugar de otro. }\end{array}$ & $\begin{array}{l}\text { Percentil de rendimiento según } \\
\text { edad cognitiva }\end{array}$ \\
\hline a. Gestos totales & Gestos deícticos y simbólicos. & \\
\hline b. Gesto tempranos & $\begin{array}{l}\text { Gestos deícticos y algunos comunicativos tempranos: "Dar"; "Mostrar"; } \\
\text { "Indicar con dedo índice"; "Decir no con la cabeza". }\end{array}$ & \\
\hline c. Gestos tardíos & $\begin{array}{l}\text { Gestos simbólicos: "peinarse con el peine"; "Dar de comer a la muñeca"; } \\
\text { "Hablar con teléfono con el zapato". }\end{array}$ & \\
\hline
\end{tabular}

*Adaptado por Galeote en una única lista de vocabulario y agregando la evaluación de "comprende y gesticula". 
el cuidador participante en los talleres, previo al inicio del "Taller para padres" (tiempo $0=\mathrm{t} 0$ ), y su resultado se mantuvo ciego para las fonoaudiólogas instructoras que impartían los talleres. Además, se registraron los antecedentes demográficos de los participantes, antecedentes mórbidos, número de hermanos, edad y nivel académico de ambos padres, asistencia a sala cuna/ jardín infantil y asistencia a programa de estimulación temprana.

2: Capacitación en comunicación gestual: se realizaron capacitaciones a 7 grupos, cada uno conformado por 3 a 4 diadas (niño - cuidador significativo). Las capacitaciones se realizaron entre marzo y agosto 2017, con una estructura según la metodología de "Señas, palabras y juegos”, del Programa Baby Signs ${ }^{\circledR 20}$. El cuidador significativo fue considerado aquel padre, madre o cuidador que permanece con el niño al menos 10 horas semanales.

Las capacitaciones consistieron en 7 talleres presenciales conducidos por dos fonoaudiólogas certificadas como instructoras en comunicación gestual por metodología Baby Signs ${ }^{\circledR}$. El primero fue un "Taller para padres" donde se les enseñó las bases de la comunicación por gestos, y los siguientes 6 talleres consistieron en una clase semanal de 60 minutos de duración, de acuerdo a la metodología "Señas, palabras y juegos" Baby Signs ${ }^{\circledR}$, abordando los siguientes temas: comer, dormir, vestirse, bañarse, mascotas y el parque. A cada participante se le entregó material consistente en cuatro libros para trabajar los gestos, una caja de tarjetas de gestos, un CD de cancionero, y un CD de cada tema con información para la comunicación gestual, para que pudiesen practicar en sus casas.

3. Evaluación final: posterior a tres meses de finalizados los talleres se les aplicó nuevamente el CDI- SD (tiempo final $=\mathrm{tf}$ ) a través de una plataforma online. Para evaluar las respuestas según edad cognitiva, se estimó la edad cognitiva del tf usando un ajuste proporcional considerando edad cronológica en $\mathrm{t} 0 \mathrm{y}$ tf $\mathrm{y}$ edad cognitiva en t0 calculada según Test de Bayley, usando la siguiente fórmula:

\section{Edad cognitiva $\mathrm{tf}=($ edad cognitiva $\mathrm{t} 0 \mathrm{x}$ edad cronológica tf) / edad cronológica t 0 .}

Al finalizar el proceso, se les solicitó a los cuidadores que completaran una encuesta sobre satisfacción y percepción personal en relación a las capacitaciones, que se basó en dos preguntas abiertas 1. Beneficio que obtuvo el niño al participar en los talles; 2. Beneficio que obtuvo usted (el cuidador), al participar en los talleres.

\section{Análisis de resultados}

Para cada niño se compararon los resultados obtenidos en el CDI-SD previo a la intervención (t0) y tres meses posterior a ella (tf). Se denominó "cambio de percentil" a la diferencia entre el percentil obtenido en el tf y el percentil obtenido en el t0. Cambio de percentil positivo correspondió al aumento de más de 5 percentiles (mejora) posterior a la intervención, sin cambio de percentil, correspondió a resultados similares pre y post intervención, con un delta de percentiles menor o igual a 5, y cambio de percentil negativo correspondió a disminución de resultados posterior a la intervención, con una disminución mayor a 5 percentiles.

La encuesta de satisfacción fue descrita como "citas" de las respuestas y comentarios de los cuidadores.

\section{Consideraciones éticas}

Se solicitó la firma de un consentimiento informado a los padres de los participantes y se contó con la aprobación del Comité de Ética de Investigación de la Facultad de Medicina de la Pontificia Universidad Católica de Chile.

El estudio fue financiado por el concurso Semilla de SOCHIPE 2016.

\section{Resultados}

Cuarenta y nueve niños entre 22 y 30 meses de edad cronológica contestaron la invitación al proyecto, a los cuales se les realizó el Test de Bayley III para determinar su edad cognitiva. De ellos, 15 tenían edad cognitiva de 17 meses o menos, 30 entre 18 y 22 meses y 4 tenían una edad cognitiva mayor a 22 meses. El grupo objetivo fue conformado por 24 niños con edad cognitiva entre 18 y 22 meses ( 6 niños con edad cognitiva objetivo no pudieron participar en las capacitaciones debido al horario de los talleres).

A los 24 niños seleccionados se les invitó a las capacitaciones en comunicación gestual. Un participante fue excluido por no asistir al "Taller para padres", requisito para participar en las capacitaciones. De los 23 participantes, 21 completaron más del $80 \%$ de asistencia a los talleres.

De los 21 niños que completaron los talleres, y de acuerdo a los resultados del Test de Bayley III aplicado a ellos, el 29\% tenía desarrollo cognitivo normal y 71\% retraso leve del desarrollo; ningún participante presentó retraso moderado ni severo. En cuanto al lenguaje, 5\% presentó desarrollo normal, 52\% retraso leve y $43 \%$ retraso moderado del desarrollo del lenguaje.

Del total de participantes que completaron los talleres, 11/21 (52\%) eran de sexo masculino, con un promedio de edad cronológica de 27,5 ( $\mathrm{DE} \pm 2,5)$ meses y 19,8 (DE $\pm 1,0)$ meses de edad cognitiva. Los antecedentes demográficos del grupo intervenido se resumen en la tabla 2 . 
Posterior a la intervención, 29\% de los participantes tuvo un cambio de percentil positivo en el ítem "comprensión de primeras frases" del CDI-SD, 52\% no tuvo cambio de percentil y en el 19\% de ellos tuvo un cambio de percentil negativo posterior a la intervención, el $62 \%$ cambió positivamente de percentil en la producción de vocabulario con gestos, el 33\% mejora en percentiles en la comprensión de vocabulario,

Tabla 2. Características demográficas de los participantes

\begin{tabular}{|c|c|}
\hline Variable & $\mathrm{n}(\%)$ \\
\hline Total de participantes & 21 \\
\hline Sexo masculino & $11(52)$ \\
\hline Edad cronológica en meses, promedio $\pm \mathrm{DE}$ & $27,5 \pm 2,5$ \\
\hline $\begin{array}{l}\text { Edad mental en meses (según Bayley III), promedio } \pm \text { DE } \\
-18-19 \\
-20-21 \\
-22\end{array}$ & $\begin{array}{l}19,8 \pm 1,0 \\
9(43) \\
11(52) \\
1 \quad(5)\end{array}$ \\
\hline $\begin{array}{l}\text { Comorbilidades } \\
\text { - Cardiopatía congénita* } \\
\text { - Alteraciones otorrinolaringológicas en tratamiento }{ }^{\dagger} \\
\text { - Hipotiroidismo en tratamiento } \\
\text { - } \text { Otras }^{\ddagger}\end{array}$ & $\begin{array}{l}9(43) \\
3(14) \\
3(14) \\
8(38)\end{array}$ \\
\hline $\begin{array}{l}\text { Hermanos } \\
-0 \\
-\geq 1\end{array}$ & $\begin{array}{r}7(33) \\
14(67)\end{array}$ \\
\hline Primogénito & $7(33)$ \\
\hline Asistencia a centro escolarizado ${ }^{\S}$ & $14(67)$ \\
\hline Asistencia a centro de estimulación temprana & $17(81)$ \\
\hline $\begin{array}{l}\text { Edad materna en años } \\
-<35 \\
-\geq 35\end{array}$ & $\begin{array}{r}2(10) \\
19(90)\end{array}$ \\
\hline $\begin{array}{l}\text { Cuidador principal que participó en talleres } \\
\text { - Mamá } \\
\text { - Papá } \\
\text { - Otro }\end{array}$ & $\begin{array}{r}18(85) \\
2(10) \\
1 \quad(5)\end{array}$ \\
\hline $\begin{array}{l}\text { Nivel educacional mamá } \\
\text { - Escolar incompleta } \\
\text { - Escolar completa } \\
\text { - Técnico- Superior }\end{array}$ & $\begin{array}{rr}0 & (0) \\
1 & (5) \\
20 & (95)\end{array}$ \\
\hline $\begin{array}{l}\text { Nivel educacional papá } \\
\text { - Escolar incompleta } \\
\text { - Escolar completa } \\
\text { - Técnico- Superior }\end{array}$ & $\begin{array}{rr}1 & (5) \\
0 & (0) \\
18 & (85)\end{array}$ \\
\hline $\begin{array}{l}\text { Asistencia a talleres } \\
->80 \% \\
-\leq 80 \%\end{array}$ & $\begin{array}{r}18(86) \\
3(14)\end{array}$ \\
\hline
\end{tabular}

DE: Desviación Estándar. *Comunicación interauricular operada; Ductus arterioso persistente hemodinamicamente significativo cerrado; Canal autrículo ventricular operado. 'Uso de tubos de ventilación; Estrechez de conducto auditivo, Hipoacusia en tratamiento con audífono desde los 6 meses de vida. 'Estenosis subglótica operada; Estenosis esofágica operada; deficit de hormona de crecimiento en tratamiento; Tiroiditis de Hashimoto; Síndrome Mieloproliferativo; Epilepsia en tratamiento; Miopía. ${ }^{\S}$ Sala cuna; Jardín infantil. ${ }^{\sharp} \mathrm{Al}$ momento del nacimiento de su hijo. el 57\% disminuyó su percentil en gestos tempranos y el $43 \%$ tuvo un cambio positivo de percentiles en la producción de gestos tardíos. Sólo 3 (14\%) niños cambiaron su percentil en forma positiva en relación a la producción de vocabulario hablado. La tabla 3 resume el rendimiento individual en percentiles de los items "Comprensión de las primeras frases" y "Vocabulario: comprensión, producción y vocabulario con gestos", y "Gestos: totales, tempranos y tardíos" del CDI-SD, y hace referencia al delta de percentiles individuales, destacando en colores la variación individual de rendimiento, donde rojo significa delta de cambio de percentil negativo, amarillo sin delta de cambio de percentiles, y verde, delta de cambio de percentil positivo.

Los resultados grupales expresados en promedio de porcentajes de respuestas positivas de los ítems $1 \mathrm{~A}, 1 \mathrm{C}$ y 1 E se muestran en la tabla 4.

En la evaluación subjetiva, todos los cuidadores refirieron beneficios en relación a la capacitación, las citas que con mayor frecuencia se reportaron se resumen en la tabla 5, refiriendo beneficios principalmente respecto al cambio en las habilidades de la comunicación de los niños, disminución de ansiedad, como también una mejoría en la comunicación entre padres e hijos, mejor capacidad de entenderlos y tranquilidad en saber qué quieren expresar.

\section{Discusión}

El presente estudio muestra que las capacitaciones en comunicación gestual de forma sistematizada favorecen el desarrollo de habilidades comunicativas en un grupo de niños con SD, principalmente en la comprensión del lenguaje y en la producción de gestos a 3 meses de seguimiento, observándose cambios positivos en el percentil de trayectoria de desarrollo de habilidades comunicativas.

Los resultados describen características del desarrollo comunicativo en niños con $\mathrm{SD}$, que ponen de manifiesto tendencias ya descritas previamente en la literatura, como una mayor capacidad en la comprensión versus la expresión, lo que se mantiene e incluso aumenta posterior a la intervención, apoyando lo descrito previamente por Abbeduto, donde la comprensión tendría mejor desarrollo que la expresión en los niños con $\mathrm{SD}^{8,21}$.

En cuanto a la comunicación gestual, los resultados en nuestra serie muestran que hay mayor producción de gestos totales a expensas de gestos tardíos, lo que aumenta posterior a la intervención. Estos resultados respaldan a la comunicación gestual como una herramienta complementaria para el desarrollo comunicativo de los niños con SD, ya que se observa que usan gestos de diferente complejidad aún sin producción 


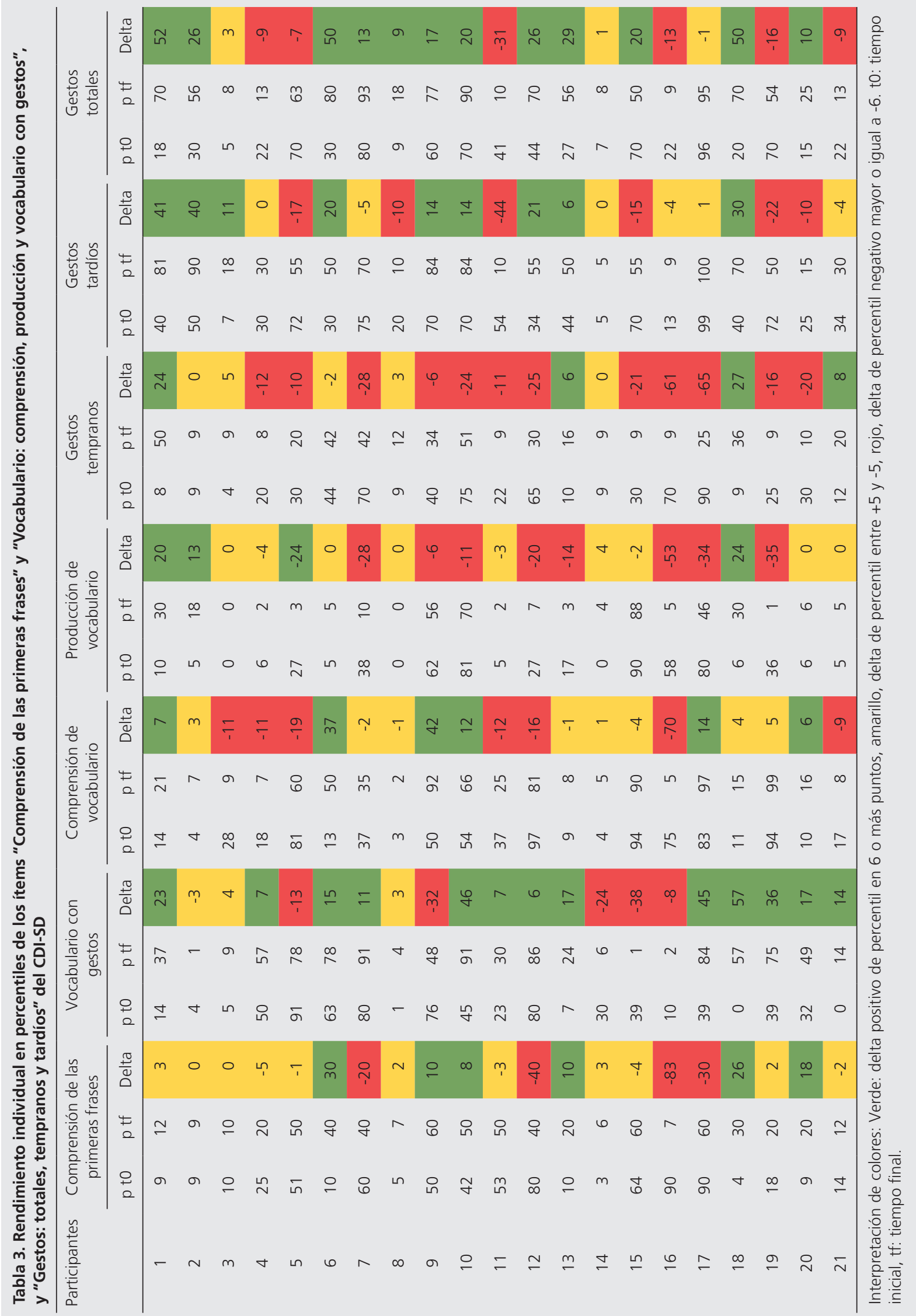


Tabla 4. Promedio grupal de porcentaje de rendimiento para "Comprensión temprana", "Inicios de la producción" y "Uso descontextualizado del lenguaje", según edad cognitiva

\begin{tabular}{|c|c|c|c|c|c|c|}
\hline & \multicolumn{2}{|c|}{$18-19(n=9)$} & \multicolumn{2}{|c|}{$20-21(n=11)$} & \multicolumn{2}{|c|}{$22-23(n=1)$} \\
\hline & $\%$ to - \% tf & $\%$ Ref* & $\%$ t0 - \% tf & $\%$ Ref* & $\%$ to $-\%$ tf & $\%$ Ref* \\
\hline \multicolumn{7}{|l|}{ Comprensión temprana } \\
\hline Responde a su nombre & $100-100$ & 100 & $90,9-90,9$ & 100 & $100-100$ & 100 \\
\hline Responde al no & $88,8-100$ & 96 & $90,9-90,9$ & 100 & $100-100$ & 100 \\
\hline Busca cuando llama mamá/papá & $100-100$ & 92 & $81,8-90,9$ & 96,7 & $0-100$ & 91,3 \\
\hline \multicolumn{7}{|l|}{ Inicios de la producción } \\
\hline Imita & $22,2-44,4$ & 52 & $54,5-45,5$ & 70 & $100-100$ & 95,7 \\
\hline Nombra & $11,1-22,2$ & 28 & $27,3-45,5$ & 56,7 & $0-100$ & 47,8 \\
\hline \multicolumn{7}{|l|}{ Uso descontextualizado del lenguaje } \\
\hline Pasado & $0-22,2$ & 12 & $9,1-9,1$ & 20 & $100-0$ & 34,8 \\
\hline Futuro & $0-11,1$ & 24 & $18,2-18,2$ & 26,7 & $0-100$ & 39,1 \\
\hline Objeto ausente (producción) & $11,1-22,2$ & 32 & $27,3-45,5$ & 60 & $100-0$ & 60,9 \\
\hline Objeto ausente (comprensión) & $33,3-66,7$ & 64 & $54,4-72,7$ & 90 & $100-0$ & 95,7 \\
\hline Dueño ausente & $33,3-22,2$ & 32 & $36,4-27,3$ & 66,7 & $100-0$ & 82,6 \\
\hline
\end{tabular}

Se muestra el promedio grupal de porcentaje inicial (\% t0) y el promedio grupal porcentaje final (\% tf) según grupo de edad mental: 18 a 19 meses, 20 a 21, y 22 meses. * \%Ref: porcentaje de respuestas positivas para dichos grupos etarios (edad cognitiva) referenciados por Galeote ${ }^{15}$.

Tabla 5. Principales apreciaciones subjetivas de los padres 3 meses posterior a los talleres

\begin{tabular}{ll}
\hline Beneficio que obtuvo su hijo al participar de este taller & Beneficio que obtuvo usted al participar de este taller \\
\hline - Sociabilización & - Inclusión familiar \\
- Intención comunicativa & - Disminución de la ansiedad \\
- Aumento de vocabulario & - Mejor comunicación \\
- Entretención con los talleres & - Entender y atender necesidades \\
- Disminución de conductas agresivas & $-\quad$ Compartir con otras duplas \\
- Vínculo emocional con otros participantes & \\
- Disminución de ansiedad & \\
\hline
\end{tabular}

de palabras, con el fin último de comunicarse ${ }^{22-24}$, lo que apoya lo descrito por Berglund, quien describe que el vocabulario productivo de niños con SD y niños con desarrollo típico son equiparables si tomamos en cuenta la producción de gestos ${ }^{25}$. En nuestro estudio, la mayoría de los niños intervenidos mantiene o aumenta su capacidad de producción de gestos totales, no así la producción de palabras, donde sólo un pequeño porcentaje aumenta esta habilidad. Esto podría deberse a que en la medida que desaparecen los gestos aparece la producción oral, lo que no se alcanzó a observar en las edades mentales de los niños evaluados en este estudio por ser una habilidad que aparece más tardíamente y por el corto periodo de observación post intervención.

Galeote et al $^{13}$ evaluó el desarrollo del vocabulario comprensivo, y la producción oral y gestual de 230 niños con SD entre 8 y 29 meses de edad mental, donde describió una superioridad en la comprensión del vocabulario por sobre la producción oral, siendo esta última más pronunciada a mayor edad mental. En relación a los gestos, Galeote describe un aumento lento a edades mentales menores, con una estabilización alrededor de los 20 a 22 meses de edad mental y una disminución posterior. Estos resultados se asemejan a los encontrados en nuestro estudio, en el cual hay mejoras en los ítems de comprensión por sobre producción oral, con aumento de la producción gestual. Destaca el aumento de gestos totales y tardíos con disminución de los tempranos, concordante a lo descrito en la literatura $^{8}$, donde los gestos tempranos típicamente van desapareciendo en la medida que aumentan los gestos tardíos. Así mismo, la literatura describe que en la medida que disminuyen los gestos totales, hay un aumento de la aparición de la palabra, lo que probablemente no se alcanzó a observar en nuestro tiempo de seguimiento. 
La observación de ausencia de cambio en los ítems "responder al nombre", "responder al no", "imitar" o "nombrar", se debe a que son habilidades ya adquiridas en el t0 y se mantienen al final del proceso. Al comparar nuestros resultados con los porcentajes referenciales de Galeote $^{18}$, los grupos tienen un rendimiento similar en el ítem de comprensión temprana, sin embargo, en el uso descontextualizado del lenguaje, hay un rendimiento de nuestro grupo por debajo de la referencia de Galeote, aunque mejora posterior a la intervención. Las diferencias entre el grupo de Galeote y el nuestro pudiese estar atribuido a elementos de selección y tamaño muestral, así como a la comprensión del castellano español, versus el castellano chileno de los padres que contestaron el CDI-SD.

Los resultados de la presente serie muestran una gran variabilidad interindividual, reflejada en la Tabla 3 , la que está descrita no tan solo en niños con SD, sino que también en niños con desarrollo típico.

Cabe destacar que un porcentaje de niños reduce su rendimiento en algunas dimensiones, en especial en la producción y comprensión de vocabulario, lo que pudiese estar determinado por la herramienta utilizada (CDI-SD), la que corresponde a un auto-reporte realizado por los padres, lo que requiere de observación por parte de ellos para tener una evaluación fidedigna del comportamiento de sus hijos. En este contexto, los papás, al no estar familiarizados previamente con el inventario CDI-SD y haberlo contestado en la primera sesión de las capacitaciones, contestaron sin observación intencionada de sus hijos, sino que con la representación que tenían de ellos, lo que podría generar un sesgo por sobrevaloración de las habilidades ${ }^{19}$, lo que podría inferirse en especial en algunos niños que cuentan con puntajes máximos en algunas dimensiones al inicio del estudio (participantes 5 y 16 de la Tabla 3). Por otro lado, el CDI-SD es un inventario largo, que requiere de un periodo prolongado de atención (alrededor de 60 a $90 \mathrm{~min}$ ), lo que puede llevar a pérdida de interés en el instrumento, y disminución en la veracidad de las respuestas. Es conocido que la producción de vocabulario en niños con SD suele ser más lenta de lo que se espera en sus restantes habilidades cognitivas, lo que pudiera desarrollarse mayormente después de los 36 meses $^{26}$, lo que requeriría de mayor seguimiento para observar la aparición de nuevas palabras. Por otra parte, se describen problemas en el habla inteligible, es decir, mayor frecuencia de dispraxia verbal ${ }^{27}$, lo que puede determinar dificultad en el reconocimiento de la aparición de nuevas palabras. Adicionalmente, está descrito que hasta un $16 \%$ de los niños con SD pudiesen desarrollar trastorno del espectro autista y afectación de la comunicación expresiva ${ }^{28}$. Ambas condiciones pudieran influir en el desarrollo de vocabulario, no así el desarrollo de gestos. Lamentablemente, el tiempo de observación de nuestro estudio no permitió detectar a niños con dispraxia o con trastorno del espectro autista, como para atribuir la disminución de producción de vocabulario a dichas condiciones. Finalmente, no pareciera que la intervención realizada pudiera producir un retroceso en las habilidades previamente adquiridas en el grupo estudiado, sin embargo, el análisis debe hacerse caso a caso.

Dentro de las limitaciones del estudio consideramos que si bien el CDI-SD es el mejor instrumento disponible en español y validado para niños con $\mathrm{SD}$, no está validado en población chilena, lo que pudiese generar dificultad en la comprensión por parte de los cuidadores, respecto a algunas palabras del instrumento. El presente reporte tiene un tamaño muestral pequeño que sólo permite describir niño a niño para generar una impresión individualizada, más que valores grupales. El seguimiento a corto plazo de los participantes, pudo haber subestimado el impacto de la intervención, por lo que sería de gran interés poder realizar un seguimiento a largo plazo y determinar el impacto en la generación de palabras en subgrupo de niños.

Tomando en cuenta los resultados de nuestra serie, consideramos que las capacitaciones en comunicación gestual son una herramienta complementaria y valiosa para la estimulación del desarrollo de niños con SD. En los casos donde se obtuvo mayor tendencia de resultados negativos, se pudo identificar dificultades en el uso del inventario CDI-SD o alteración del desarrollo social, lo que pudo haber interferido con la adquisición de nuevas habilidades. Considerando las limitantes del CDI-SD, sería adecuado instruir a los cuidadores que van a participar de las capacitaciones, sobre los aspectos que se van a evaluar en el CDI-SD, de manera de ser observados y registrados en forma más objetiva.

\section{Conclusiones}

El presente es el primer estudio latinoamericano que describe la respuesta de un grupo de niños con SD a una técnica de enseñanza en comunicación gestual.

El grupo de niños con SD es un grupo heterogéneo, a pesar de homogeneizarlos según su edad cognitiva, se observa una variabilidad interindividual que no permite extrapolar los resultados a grupos ni a toda la población de niños con SD.

Considerando que la metodología de "Señas, palabras y juegos" de Baby Signs ${ }^{\circledR}$, es una estrategia que no pone en riesgo a los niños, que es de bajo costo y de fácil adquisición y reproducción por parte de los padres, y teniendo en cuenta los resultados que muestran que algunos niños podrían beneficiarse mejorando sus habilidades de comunicación, la recomendación de capacitación en comunicación gestual pudiera ser una 
estrategia de estimulación de la comunicación complementaria, considerando siempre la necesidad de realizar evaluaciones individuales y de comparación sobre sí mismos, de manera de ir evaluando progresos y eventual cambio en su propia trayectoria de desarrollo.

Se necesitan estudios de mayor tamaño muestral, con instrumentos validados en población chilena, con seguimiento de participantes a largo plazo, para evaluar el impacto en su desarrollo posterior, así como también comparación de los resultados con niños con desarrollo típico.

\section{Responsabilidades Éticas}

Protección de personas y animales: Los autores declaran que los procedimientos seguidos se conformaron a las normas éticas del comité de experimentación humana responsable y de acuerdo con la Asociación Médica Mundial y la Declaración de Helsinki.

Confidencialidad de los datos: Los autores declaran que han seguido los protocolos de su centro de trabajo sobre la publicación de datos de pacientes.
Derecho a la privacidad y consentimiento informado: Los autores han obtenido el consentimiento informado de los pacientes y/o sujetos referidos en el artículo. Este documento obra en poder del autor de correspondencia.

\section{Conflicto de intereses}

Los autores declaran no tener conflicto de intereses.

\section{Agradecimientos}

Agradecimientos a Baby Signs ${ }^{\circledR}$ Chile por el aporte de material para llevar a cabo los talleres de comunicación gestual, y a Miguel Galeote por la autorización para el uso y aplicación de Test CDI-SD.

\section{Financiamiento}

Concurso Semilla de SOCHIPE 2016.

\section{Referencias}

1. Farkas C. Comunicación Gestual en la Infancia Temprana: Una Revisión de su Desarrollo, Relación con el Lenguaje e Implicancias de su Intervención. Psykhe 2007;16(2):107-15

2. Acredolo L. Goodwyn S, Symbolic gesturing in normal infants. Child Dev 1988;59:450-99.

3. Farkas C. Fomentando gestos simbólicos en infantes: impacto sobre el estrés y la autoeficacia materna. Revista de investigación en Logopedia 2012;2: 15-37.

4. Goodwyn S, Acredolo L. Impact of symbolic gesturing on early language development, J Nonverbal Behav 2000; 24 (2):81-103

5. Acredolo L, Goodwyn S. How to build a baby that can read minds: Cognitive mechanisms in mindreading, Curr Psychol Cogn 2001;13(5):513-52.

6. Vallotton C. Signs of emotion: What can preverbal children "say" about internal states? Infant Ment Health J 2008;29(3):234-58

7. Acredolo L, Goodwyn S. The long-term impact of symbolic gesturing during infancy on IQ at age 8 (dissertation). Brighton (UK): International conference on infant studies, 2000.

8. Abbeduto L. Language Development in Down syndrome form the prelinguistic period to acquisition of literacy, Ment
Retard Dev Disabil Res Rev 2007;13:24761.

9. Galeote M, Soto P, Checa E, et al. The acquisition of productive vocabulary in Spanish children with Down síndrome. J Intellect Dev Disabil. 2008;33(4):292302.

10. Caselli MC, Vicari S, Longobardi E, et al. Gestures and words in early development of children with Down syndrom, J Speech Lang Hear Res. 1998;41(5):1125-35.

11. Clibbens J. Signing and Lexical Development in Children with Down Syndrome, Downs Syndr Res Pract 2001; 7(3):101-5.

12. Mainela-Arnold E, Gesture-speech integration in children with specific language impairmet, Int J Lang Commun Disord. 2014;49(6):761-70.

13. Galeote M, Sebastián E, Checa E, et al. The development of vocabulary in Spanish children with Down syndrome: comprehension, production, and gestures, J Intellect Dev Disabil. 2011;36(3):184-96.

14. Liszkowski U, Brown P, Callaghan T, et al. A prelinguistic gestural universal of human communication. Cogn Sci. 2012;36(4):698-713.

15. Jackson-Maldonado D, Bates E, Thal D. MacArthur Inventario del Desarrollo de Habilidades Comunicativas (Inventario). 2003, Baltimore: Brookes Publishing.

16. Galeote M, Checa E, Sánchez-Palacios C, et al. Adaptation of the MacArthur-Bates Communicative Development Inventories for Spanish children with Down Syndrome: Validity and reliability data for vocabulary, American journal of speech-language pathology 2016;25(3):371-80.

17. Bayley N, Bayley Scales of Infant and Toddler Development, J Psychoeduc Assess. 2007;25(2):180-98.

18. Galeote $\mathrm{M}$ (manuscrito sin publicar). Inventario de Desarrollo Comunicativo MacArthur-Bates adaptado a niños con síndrome de Down. Universidad de Málaga: Departamento de Psicología Evolutiva y de la Educación, http:// webpersonal.uma.es/ mgaleote/, última visita 05.07.2018.

19. Galeote M, Soto P, Serrano A, et al. Un nuevo instrumento para evaluar el desarrollo comunicativo y lingüístico de niños con síndrome de Down, Revista Síndrome de Down. 2006;23,1(88): 20-6.

20. Farkas C. Actividades para el uso de los gestos, En: Gestos que hablan: aprendiendo a comunicarnos con nuestros niños, Ed. Universidad Católica de Chile, Santiago, 2009; 42-8.

21. Chan J, Iacono T. Gesture and word production in children with Down síndrome, Augment Altern 2001;73-87.

22. Dimitrova N. Parents'Translations of Child Gesture Facilitate Word Learning in Children with Autism, Down Syndrome and Typical Development, J Autism Dev Disord 2016;46(1):221-31. 
23. Ózcaliskan S. Baby sign but not spontaneous gesture predicts later vocabulary in children with Down Syndrom. J Child Lang. 2016;43(4):94863.

24. Özçalışkan Ş, Adamson LB, Dimitrova $\mathrm{N}$, Baumann S. Early gesture provides a helping hand to spoken vocabulary development for children with autism, Down syndrome and typical development.
J Cogn Dev. 2017;18(3):325-37.

25. Berglund E, Parental reports of spoken language skills in children with Down syndrome, J Speech Lang Hear Res. 2001; 44:179-91.

26. Zampini L, D’Odorico L. Vocabulary development in children with Down syndrome: longitudinal and crosssectional data. J Intellect Dev Disabil. 2013;38(4):310-7.
27. Kumin L. Speech intelligibility and childhood verbal apraxia in children with Down syndrome. Downs Syndr Res Pract. 2006;10(1):10-22.

28. Richards C, Jones C, Groves L, Moss J, Oliver C. Prevalence of autism spectrum disorder phenomenology in genetic disorders: a systematic review and meta-analysis. Lancet Psychiatry. 2015;2(10):909-16. 